\title{
Energy absorption by the human body from RF and microwave emissions in Sri Lanka
}

\author{
M. A. A. Karunarathna and I. J. Dayawansa*
}

Department of Electronic and Telecommunication Engineering, University of Moratuwa, Moratuwa

\begin{abstract}
This paper presents the computed Specific Absorption Rate (SAR) in the human eye using measured field strengths from RF signals radiated by FM radio, TV and mobile cellular base stations operating in the $900 \mathrm{MHz}$ band, in different parts of Sri Lanka. The SAR values in the eye are well below the internationally recognized FCC standards on human exposure to RF radiation. Calculations were carried out using the Finite Difference Time Domain (FDTD) method.

The estimated SAR distribution in some organs of the human body due to exposure from signals of frequencies ranging from $100 \mathrm{MHz}$ to $2500 \mathrm{MHz}$, based on an incident field of strength $1 \mathrm{~V} / \mathrm{m}$, are also presented. Some of the most vital organs of the human body, the eye, the testis, the brain and the kidney, have been examined. Results indicate that in all the organs investigated except in the testis, the maximum SAR increases with increasing frequency. Among the four organs the highest SAR of value $0.308 \mathrm{~W} / \mathrm{kg}$ is in the eye and it occur at the highest frequency.

The SAR distribution in the eye shows that beyond $900 \mathrm{MHz}$ its maximum value shifts towards the interior of the eye and at $2500 \mathrm{MHz}$ it is almost at the centre of the eye. In the kidney, at low frequencies, the highest SAR is at the front layers and it gets shifted to the centre of the kidney at 900 $\mathrm{MHz}$ and beyond this frequency the peak SAR shifts towards the interior. SAR values inside the four vital human body organs analyzed in this paper are below FCC standards and therefore at present we are safe.
\end{abstract}

\section{INTRODUCTION}

The rapid deployment of wireless communication systems such as cellular phones has caused an increased concern for the potential dangers to public health as a result of exposure to electromagnetic waves. Signals are transmitted in the form of electromagnetic waves in all directions by FM radio transmitters broadcasting in the VHF region and TV transmitters broadcasting in the VHF and UHF

\footnotetext{
*Corresponding Author, E-mail: indra@ent.mrt.ac.lk
} 
regions. In cellular mobile systems and fixed wireless local loop systems, base stations transmit in the UHF and microwave regions. Computer networks and the Internet are accessed using wireless techniques at frequencies in the microwave range from 2 to $4 \mathrm{GHz}$, using bluetooth technology for short distances, WiFi technology for longer distances and WiMax technology for very long distances such as 10 to $20 \mathrm{~km}$. As the number of users grows, more base stations are installed to increase the system capacity. In urban areas, base stations are closer together but operated at lower power levels than the rural areas where cells tend to be larger in size. Thus we are constantly being exposed to RF and microwave radiation from a variety of wireless communication systems. The electromagnetic waves of different power levels and different frequencies penetrate into the human body causing health risks; this is of great public concern.

The rate at which electromagnetic energy is absorbed by the tissues of the human body is usually quantified by the specific absorption rate or the SAR value. It is a quantity that depends on the tissue mass and the strength of the electromagnetic waves inside the body as given in equation (1),

$$
S A R=\frac{1}{2} \frac{\sigma}{\rho}|E|^{2}
$$

where $E$ is the electric field intensity of the electromagnetic wave in the tissue, $s$ and $r$ are the conductivity and tissue mass respectively, of the human body organ under examination. To avoid adverse health effects, several reputed organizations such as the Institute of Electrical and Electronic Engineers [1] (IEEE), Federal Communications Commission [2] (FCC), the National Council on Radiation Protection and Measurements [3] (NCRP) and the International Committee on NonIonizing Radiation Protection [4] (ICNIRP) and the National Radiation Protection Board [5] (NRPB) have adopted exposure guidelines for the general public as well as for RF workers in the course of their regular duties. According to FCC standards, the safe level of SAR for the general public is 1.6 $\mathrm{W} / \mathrm{Kg}$ in $1 \mathrm{~g}$ of tissue. Standards also specify the exposure level in terms of the incident electric field strength, $E(\mathrm{~V} / \mathrm{m})$, and the power density, $S\left(\mathrm{~W} / \mathrm{m}^{2}\right)$. 'Exposure Quotient' is a useful dimensionless quantity that expresses the exposure due to electric fields of multiple radio signals at any location as given in equation (2).

$$
\text { Exposure..Quotient }=\sum_{i=1}^{n} \frac{S_{i}}{M P E_{i}}
$$

where $S_{i}$ is the power density and $M P E$ is the maximum permissible exposure of power density at the $i^{\text {th }}$ frequency. For safety, the 'exposure quotient' must be less than or equal to one.

\section{BIOLOGICAL EFFECTS DUE TO EXPOSURE}

It has been known for many years that exposure to RF radiation can be harmful due to the ability of RF energy to heat biological tissue rapidly. Exposure to very high RF power densities of the order of 
$100 \mathrm{~mW} / \mathrm{cm}^{2}$, can result in heating biological tissues and thereby increasing the body temperature [6]. Tissue damage could also occur during exposure to high RF levels because the body is unable to cope with or dissipate the excessive heat that could be generated. Under certain conditions, exposure to RF energy at power density levels of 1 to $10 \mathrm{~mW} / \mathrm{cm}^{2}$ can result in measurable heating of biological tissue [6] but not necessarily cause tissue damage. Two areas of the body, the eyes and testis, are known to be particularly susceptible to heating by RF energy because of the relative lack of available blood flow to dissipate the excessive heat. It is reported that short term exposure to very high levels of RF radiation have caused cataracts in rabbits [6]. Temporary sterility, caused by such effects as changes in sperm count and sperm mobility, is reported to be possible after exposure of the testis to high-level RF radiation. Mice and rabbits have been employed for most of the experimental investigations on biological effects of RF exposure.

The RF exposure levels in different parts of Sri Lanka are known [7]. The measured values consisted of fields radiated by a large number of FM radio, TV and cellular mobile base stations. From several spot measurements taken from over 20 major towns in the country it has been shown that the exposure levels at these locations are well below FCC standards [2,7]. This paper reports the SAR values calculated for the human eye, using the measured values of the electric field strength in different cities in Sri Lanka and they are compared with the FCC standards. The eye is taken for the calculations because it is one of the most sensitive organs of the human body. This paper also reports the results of estimated SAR distribution in different organs of the human body such as the eye, brain, kidney and testis, due to different incident RF signals at different frequencies.

\section{FIELD DISTRIBUTION INSIDE THE BODY AND SAR CALCULATIONS}

Several methods have been described in the literature [8] for numerical calculations of rates of electromagnetic energy absorption. One common technique is the Method Of Moments or MOM which is a frequency domain technique. The other common method is the Finite Difference Time Domain or FDTD which is a time domain method. The FDTD method first proposed by Yee [9] and later developed by many researchers [10-12] is the most often used numerical technique to solve the electromagnetic dosimetry problems [13-15]. This paper reports results of SAR calculations using FDTD method to examine the SAR distribution in different organs of the human body.

\subsection{Field Distribution inside the body}

The electric field intensity at different points that make up the organ under investigation is obtained using the FDTD method. In this method, Maxwell's equations in the differential form are solved when an incident uniform plane electromagnetic wave propagates through the body as shown in 
Fig.1. This is carried out in a time-stepped manner until convergence is reached.

The Maxwell's time-dependent curl equations are,

$$
\begin{aligned}
& \nabla \wedge E=-\mu \frac{\partial H}{\partial t} \\
& \nabla \wedge H=\sigma E+\varepsilon \frac{\partial E}{\partial t}
\end{aligned}
$$

The body under investigation is divided into a large number of small cubic cells of size $\delta_{\mathrm{x}}, \delta_{\mathrm{y}}, \delta_{\mathrm{z}}$ and the investigation is carried out at ' $\delta \mathrm{t}$ ' time intervals until steady state is reached.

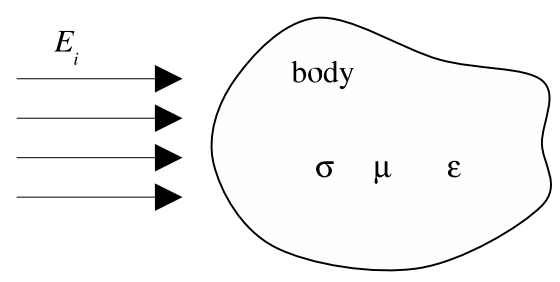

Fig. 1 Uniform plane wave incident on the body.

The components of $E$ and $H$ fields are positioned at half step intervals around a unit cell. The parameters $\sigma, \mu$ and $\varepsilon$ are the conductivity, permeability and permittivity respectively of the body. The vector equations (3) \& (4) represent a system of six scalar equations, which can be expressed in the rectangular coordinate system $(\mathrm{x}, \mathrm{y}, \mathrm{z})$ as;

$$
\begin{aligned}
& \frac{\partial E_{x}}{\partial t}=\frac{1}{\varepsilon}\left(\frac{\partial H_{z}}{\partial y}-\frac{\partial H_{y}}{\partial z}-\sigma E_{x}\right) \\
& \frac{\partial E_{y}}{\partial t}=\frac{1}{\varepsilon}\left(\frac{\partial H_{x}}{\partial z}-\frac{\partial H_{z}}{\partial x}-\sigma E_{y}\right) \\
& \frac{\partial E_{z}}{\partial t}=\frac{1}{\varepsilon}\left(\frac{\partial H_{y}}{\partial x}-\frac{\partial H_{x}}{\partial y}-\sigma E_{z}\right) \\
& \frac{\partial H_{x}}{\partial t}=\frac{1}{\mu}\left(\frac{\partial E_{y}}{\partial z}-\frac{\partial E_{z}}{\partial y}\right) \\
& \frac{\partial H_{y}}{\partial t}=\frac{1}{\mu}\left(\frac{\partial E_{z}}{\partial x}-\frac{\partial E_{x}}{\partial z}\right) \\
& \frac{\partial H_{z}}{\partial t}=\frac{1}{\mu}\left(\frac{\partial E_{x}}{\partial y}-\frac{\partial E_{y}}{\partial x}\right)
\end{aligned}
$$

The propagation of an incident RF wave, $E_{i}$, through the body having electrical properties, $\sigma, \mu$ and $\varepsilon$ is governed by equations (5) to (10). The equations are solved using FDTD method by converting the six partial differential equations into six difference equations from which all six field components, 
$E_{x}, E_{y}, E_{z}, H_{x}, H_{y}, H_{z}$ can be obtained. Following Yee's notation, we assume that the grid points of the body are defined using $(i, j, k)$ with coordinates given as, $\left(i \delta_{x}, j \delta_{y}, k \delta_{z}\right)$, where $\delta=\delta_{x}=\delta_{y}=\delta_{z}$ is the size of the unit cubic cell.

For good accuracy in FDTD simulations, the cell dimensions should be less than one tenth of the wavelength in the medium when it has the highest permittivity.

i.e:

$$
\delta \leq 0.1 \lambda_{m}=\frac{0.1 c}{f \sqrt{\varepsilon_{r}}}
$$

For stability, the time step is given by,

$$
\delta t=\frac{\delta}{2 c}
$$

In the FDTD method, the radiation condition is not implicit and therefore as the RF wave propagates inside the body, the scattered waves must be truncated at the edge of the computing field. The truncation used is in accordance with Bayliss and Turkel [16], and it results in an error as given by equation (13).

$$
\text { Error } \approx\left[\frac{\mathrm{c}}{2 \pi f r}\right]^{2.5}
$$

where $f$ is the frequency of the incident signal, $r$ is the distance from the center of the scattering site to the edge of the computing field and $c$ is the velocity of light in free space.

Equation (13) implies that problems using lower incident frequencies may require a larger volume surrounding the body to avoid errors due to imperfect truncation conditions. Thus a computing field of approximately $5 \delta$ to $10 \delta$ is used for minimum truncation error [17].

\subsection{SAR distribution}

When the E-field distribution inside the body is estimated, the SAR distribution is also easily obtained from equation (1). The region with the maximum field corresponds to the region with maximum SAR value. Hence the maximum SAR and the SAR distribution for different body organs of different shapes and sizes having different electrical properties, $\sigma, \mu$ and $\varepsilon$ can be estimated from the E-field distribution.

\section{ORGAN MODELS FOR SAR CALCULATIONS}

For SAR calculations, the body organs under investigation are modeled in such a way that calculations can be carried out without much difficulty. Spherical models are used for the human eye [18] and testis [19] with average radii being $18.75 \mathrm{~mm}$ and $25.50 \mathrm{~mm}$ respectively. A cubical box model of 
side $20 \mathrm{~cm}$ is taken for the brain. A rectangular box model of $11 \times 6 \times 4 \mathrm{~cm}$ is taken for the kidney [19]. Tissues of brain, eye sclera, kidney and testis are taken as homogeneous. For electrical characterization of the tissues, the permittivity and conductivity data reported in Gabriel's paper [20] and their highest values in the range of frequencies of interest are taken for SAR calculations. According to equation (13), the lower incident frequency dictates a larger surrounding volume to minimize errors. Rather than using an enormous amount of computer resources, the cell size was increased at lower frequencies, without sacrificing accuracy and stability of the solutions. Using the symmetrical properties of the organs, only one fourth of the organs are used for SAR evaluation, in order to reduce the computational time.

For evaluation of the SAR in the human eye at the frequency of $900 \mathrm{MHz}$, the eye was divided into a number of cubic cells of size $\delta=1.25 \mathrm{~mm}$ and enclosed in to a rectangular box of size $39 \delta \times 39 \delta \times 39 \delta$, and lattice truncation condition was applied for the FDTD method. Fig. 2 shows one forth of the eye model at this frequency. The incident plane wave is assumed to propagate in the $+y$ direction. This wave is generated at the lattice plane $y=3 \delta$. At planes $y=0, y=39 \delta, x=\delta / 2$ and $\mathrm{z}=0$ the field components $\mathrm{E}_{\mathrm{z}}$ and $\mathrm{H}_{\mathrm{x}}$ are determined using lattice truncation conditions.
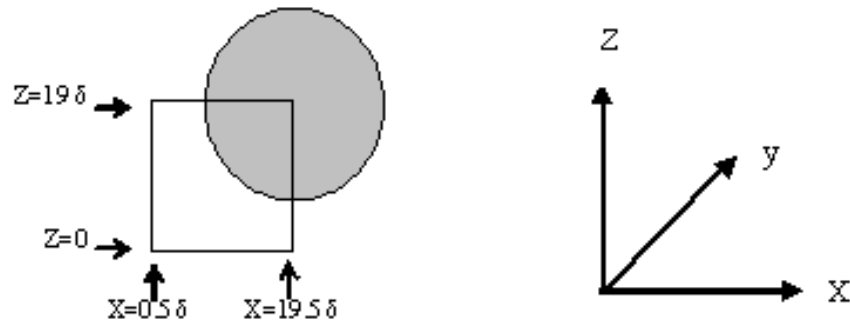

Fig. 2 The eye model and the axes

All models selected for SAR evaluation in the eye, brain, testis and kidney are shown in Fig.3.
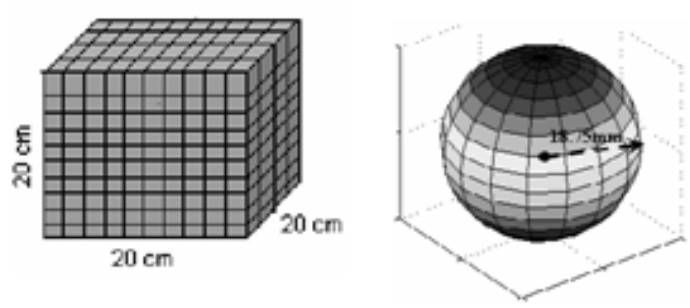

(a) cubical box model; brain (a) Spherical model; eye
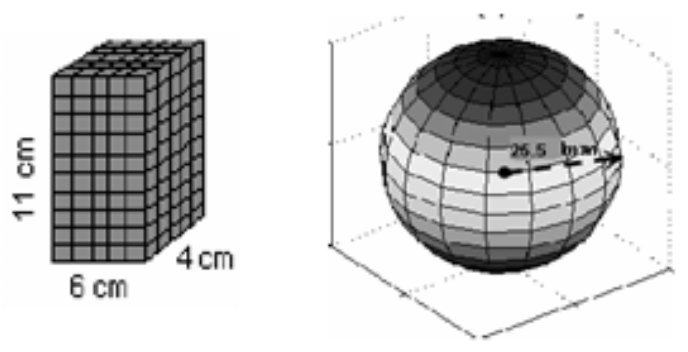

(a) rectangular box model; kidney (a) Spherical model; testis

Fig. 3 Human organ models and their dimensions (a) Brain (b) Eye (c) Kidney (d) Testis 


\section{OBSERVATIONS \& RESULTS}

\subsection{SAR Levels in the Eye of the Public}

The SAR values in the eye are evaluated using FDTD method for the public using spot field measurements [7] taken in different urban areas. As the human eye is one of the most sensitive organs of the body, the SAR values for $1 \mathrm{~g}$ tissue of the eye are evaluated for the worst-case situation for each city for compliance testing. The maximum SAR values obtained are given in Table 1. The total $\mathrm{SAR}_{1 \mathrm{~g}}$ for a particular frequency range is calculated by multiplying the maximum SAR value by the number of received signals of that frequency range. This procedure is applied to the other three frequency ranges as well and finally the grand total $\mathrm{SAR}_{1 \mathrm{~g}}$ is calculated for each site.

As seen in the results, the total $\mathrm{SAR}_{1 \mathrm{~g}}$ values in each city are well below the safe limits. The highest SAR value of $0.0922 \mathrm{~W} / \mathrm{kg}$ was observed from Badulla; measurements were made at the Uva provincial cricket grounds. The measurements indicate that the highest contributor to the SAR at Badulla is from the mobile base stations; perhaps the measurement site which is the Uva provincial

Table 1. Maximum SAR in the eye of the general public

\begin{tabular}{|c|c|c|c|c|c|c|c|c|c|c|}
\hline \multirow[t]{2}{*}{ City } & \multicolumn{5}{|c|}{ No. of received signals } & \multicolumn{5}{|c|}{$\mathrm{SAR}_{1 \mathrm{~g}}(\mathrm{~W} / \mathrm{kg})$ for the human eye } \\
\hline & $\sum_{I}$ & $\begin{array}{l}\overbrace{1}^{1} \\
z \\
z\end{array}$ & $\begin{array}{l}\text { 至 } \\
己 \\
己 \\
\end{array}$ & 音 & हैँ & $\sum_{I I}$ & 己至 & 挋 & 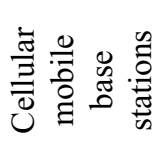 & ڤ્⿹ \\
\hline Colombo Fort & 30 & 06 & 15 & 89 & 140 & $3.69 \times 10^{-6}$ & $2.90 \times 10^{-9}$ & $7.14 \times 10^{-5}$ & $2.22 \times 10^{-2}$ & $2.22 \times 10^{-2}$ \\
\hline Nuwara-Eliya & 30 & 04 & 04 & 44 & 82 & $1.35 \times 10^{-8}$ & $3.50 \times 10^{-6}$ & $9.08 \times 10^{-5}$ & $2.94 \times 10^{-4}$ & $3.88 \times 10^{-4}$ \\
\hline Colombo Uni. & 25 & 06 & 14 & 76 & 121 & $2.24 \times 10^{-7}$ & $2.70 \times 10^{-8}$ & $2.77 \times 10^{-4}$ & $2.62 \times 10^{-3}$ & $2.90 \times 10^{-3}$ \\
\hline Kegalle & 25 & 06 & 05 & 31 & 67 & $1.63 \times 10^{-8}$ & $1.07 \times 10^{-6}$ & $1.45 \times 10^{-7}$ & $8.55 \times 10^{-3}$ & $8.55 \times 10^{-3}$ \\
\hline Dambulla & 15 & 03 & 06 & 28 & 51 & $1.21 \times 10^{-10}$ & $1.18 \times 10^{-7}$ & $4.84 \times 10^{-7}$ & $9.68 \times 10^{-3}$ & $9.73 \times 10^{-5}$ \\
\hline Matale & 34 & 06 & 06 & 24 & 70 & $8.16 \times 10^{-10}$ & $2.59 \times 10^{-8}$ & $9.59 \times 10^{-7}$ & $4.33 \times 10^{-5}$ & $4.43 \times 10^{-5}$ \\
\hline Kuliyapitiya & 35 & 06 & 05 & 12 & 58 & $2.61 \times 10^{-10}$ & $1.38 \times 10^{-8}$ & $4.14 \times 10^{-7}$ & $2.09 \times 10^{-6}$ & $2.52 \times 10^{-6}$ \\
\hline Kandy & 32 & 09 & 12 & 51 & 104 & $6.78 \times 10^{-9}$ & $4.16 \times 10^{-9}$ & $1.41 \times 10^{-6}$ & $5.47 \times 10^{-4}$ & $5.48 \times 10^{-4}$ \\
\hline Gampaha & 37 & 06 & 04 & 30 & 77 & $4.31 \times 10^{-10}$ & $3.39 \times 10^{-9}$ & $1.13 \times 10^{-7}$ & $6.38 \times 10^{-3}$ & $6.38 \times 10^{-3}$ \\
\hline Matara & 27 & 02 & 06 & 48 & 83 & $3.44 \times 10^{-11}$ & $2.53 \times 10^{-10}$ & $4.81 \times 10^{-7}$ & $6.60 \times 10^{-4}$ & $6.61 \times 10^{-4}$ \\
\hline Galle & 22 & 01 & 03 & 31 & 57 & $7.10 \times 10^{-11}$ & $2.27 \times 10^{-11}$ & $7.15 \times 10^{-8}$ & $8.22 \times 10^{-2}$ & $8.22 \times 10^{-2}$ \\
\hline Kurunegala & 34 & 04 & - & 51 & 89 & $3.54 \times 10^{-11}$ & $1.14 \times 10^{-9}$ & - & $2.60 \times 10^{-4}$ & $2.60 \times 10^{-4}$ \\
\hline Moratu. Uni. & 41 & 06 & 13 & 44 & 104 & $1.17 \times 10^{-9}$ & $7.78 \times 10^{-9}$ & $1.91 \times 10^{-5}$ & $2.33 \times 10^{-3}$ & $2.35 \times 10^{-3}$ \\
\hline Ambalangoda & 19 & 02 & 04 & 34 & 59 & $8.31 \times 10^{-12}$ & $5.76 \times 10^{-11}$ & $1.86 \times 10^{-7}$ & $6.98 \times 10^{-4}$ & $6.98 \times 10^{-4}$ \\
\hline Anuradhapura & 29 & 05 & - & 58 & 92 & $1.57 \times 10^{-11}$ & $3.31 \times 10^{-9}$ & - & $1.74 \times 10^{-2}$ & $1.74 \times 10^{-2}$ \\
\hline Mahiyangana & 14 & 03 & - & 18 & 35 & $8.04 \times 10^{-11}$ & $9.78 \times 10^{-11}$ & - & $1.52 \times 10^{-4}$ & $1.52 \times 10^{-4}$ \\
\hline Badulla & 13 & 06 & 05 & 39 & 62 & $2.37 \times 10^{-11}$ & $1.90 \times 10^{-9}$ & $1.64 \times 10^{-4}$ & $9.21 \times 10^{-2}$ & $9.22 \times 10^{-2}$ \\
\hline Ratnapura & 19 & 02 & 02 & 32 & 55 & $1.78 \times 10^{-10}$ & $3.12 \times 10^{-10}$ & $6.26 \times 10^{-8}$ & $3.34 \times 10^{-4}$ & $3.34 \times 10^{-4}$ \\
\hline Chilaw & 33 & 06 & 01 & 32 & 72 & $2.85 \times 10^{-11}$ & $3.79 \times 10^{-10}$ & $4.24 \times 10^{-8}$ & $1.63 \times 10^{-2}$ & $1.63 \times 10^{-2}$ \\
\hline Puttlam & 31 & 06 & - & 36 & 73 & $2.19 \times 10^{-11}$ & $3.34 \times 10^{-10}$ & - & $3.88 \times 10^{-3}$ & $3.88 \times 10^{-3}$ \\
\hline Negombo & 38 & 06 & - & 41 & 85 & $3.17 \times 10^{-10}$ & $9.67 \times 10^{-9}$ & - & $9.72 \times 10^{-4}$ & $9.72 \times 10^{-4}$ \\
\hline
\end{tabular}


cricket grounds is surrounded by a significant number of mobile base stations. The estimated SAR value at the site is $5.76 \%$ of the FCC standard of $1.6 \mathrm{~W} / \mathrm{kg}$ for the general public. Results in Table 1 also show that the main exposure from all sites is due to the cellular mobile base stations. As the cellular mobile base stations are rapidly on the increase, the SAR would increase beyond the values given in the table and some public concern has to be expected as high SAR could cause health risks. There are no such field measurements or SAR calculations reported from neighbouring countries for comparison. Up to now there are very few results reported from other parts of the world [21] and they include only emissions from one or two transmitters and as such the estimated SAR values are very low.

\subsection{Maximum SAR in the human body organs: eye, brain, kidney and testis}

The maximum SAR in four vital organs of the human body due to RF \& microwave exposure from a uniform field of strength $1 \mathrm{~V} / \mathrm{m}$ is examined. This field strength is a reasonable value for worst case estimates. SAR calculations are carried out for incident signals of frequencies from $100 \mathrm{MHz}$ to 900 $\mathrm{MHz}$ in the range where field strength measurements are carried out. Calculations are extended to frequencies from $900 \mathrm{MHz}$ to $2500 \mathrm{MHz}$ which are used at present in cellular mobile systems and fixed wireless communication systems. The human body organs are modeled as mentioned earlier in this section. The SAR at a single frequency is estimated as a uniform plane sinusoidal wave of that particular frequency is made to propagate into the organ. The maximum SAR values for $1 \mathrm{~g}$ of the tissue are shown in Table 2.

Table 2. Maximum $\mathrm{SAR}_{1 \mathrm{~g}}$ values in the brain, eye, kidney and the testis

\begin{tabular}{|c|c|c|c|c|c|c|c|}
\hline \multirow{2}{*}{ Organ } & \multirow{2}{*}{ Model } & \multicolumn{5}{|c|}{ Maximum $\mathbf{S A R}_{\mathbf{1 g}}(\mathbf{W} / \mathbf{k g})$} \\
\cline { 3 - 8 } & & $\mathbf{1 0 0} \mathbf{~ M H z}$ & $\mathbf{2 0 0} \mathbf{~ M H z}$ & $\mathbf{5 0 0} \mathbf{~ M H z}$ & $\mathbf{9 0 0} \mathbf{~ M H z}$ & $\mathbf{1 8 0 0} \mathbf{M H z}$ & $\mathbf{2 5 0 0} \mathbf{~ M H z}$ \\
\hline Brain & Cubical box & $1.34 \times 10^{-5}$ & $4.77 \times 10^{-5}$ & $2.89 \times 10^{-4}$ & $7.44 \times 10^{-4}$ & $17.84 \times 10^{-3}$ & $29.96 \times 10^{-3}$ \\
\hline Eye & Spherical & $1.29 \times 10^{-6}$ & $2.09 \times 10^{-5}$ & $1.27 \times 10^{-3}$ & $2.03 \times 10^{-2}$ & $11.14 \times 10^{-2}$ & $30.80 \times 10^{-2}$ \\
\hline Kidney & $\begin{array}{c}\text { Rectangular } \\
\text { box }\end{array}$ & $4.59 \times 10^{-6}$ & $2.70 \times 10^{-5}$ & $4.29 \times 10^{-3}$ & $1.16 \times 10^{-2}$ & $2.01 \times 10^{-2}$ & $6.82 \times 10^{-2}$ \\
\hline Testis & Spherical & $3.49 \times 10^{-6}$ & $6.62 \times 10^{-5}$ & $3.34 \times 10^{-3}$ & $3.17 \times 10^{-2}$ & $19.48 \times 10^{-2}$ & $15.33 \times 10^{-2}$ \\
\hline
\end{tabular}

Table 2 shows that in all organs except the testis, the maximum $\mathrm{SAR}_{\mathrm{gg}}$ values increase with increasing frequency. Our calculations show that the highest SAR is in the eye and it is approximately $0.31 \mathrm{~W} /$ $\mathrm{kg}$ and occurs at the highest frequency of $2500 \mathrm{MHz}$. The FCC standard is $1.6 \mathrm{~W} / \mathrm{kg}$. Such high values of SAR are considered to be a possible cause of eye cataract [22-23]. The SAR in the brain is higher than that of all other organs at low frequencies. In the case of the testis, the maximum SAR increases with increasing frequency; reaches a peak at $1800 \mathrm{MHz}$ and falls off as the frequency continues to increase. This is clearly seen when the SAR distribution is examined as discussed in the next section. 

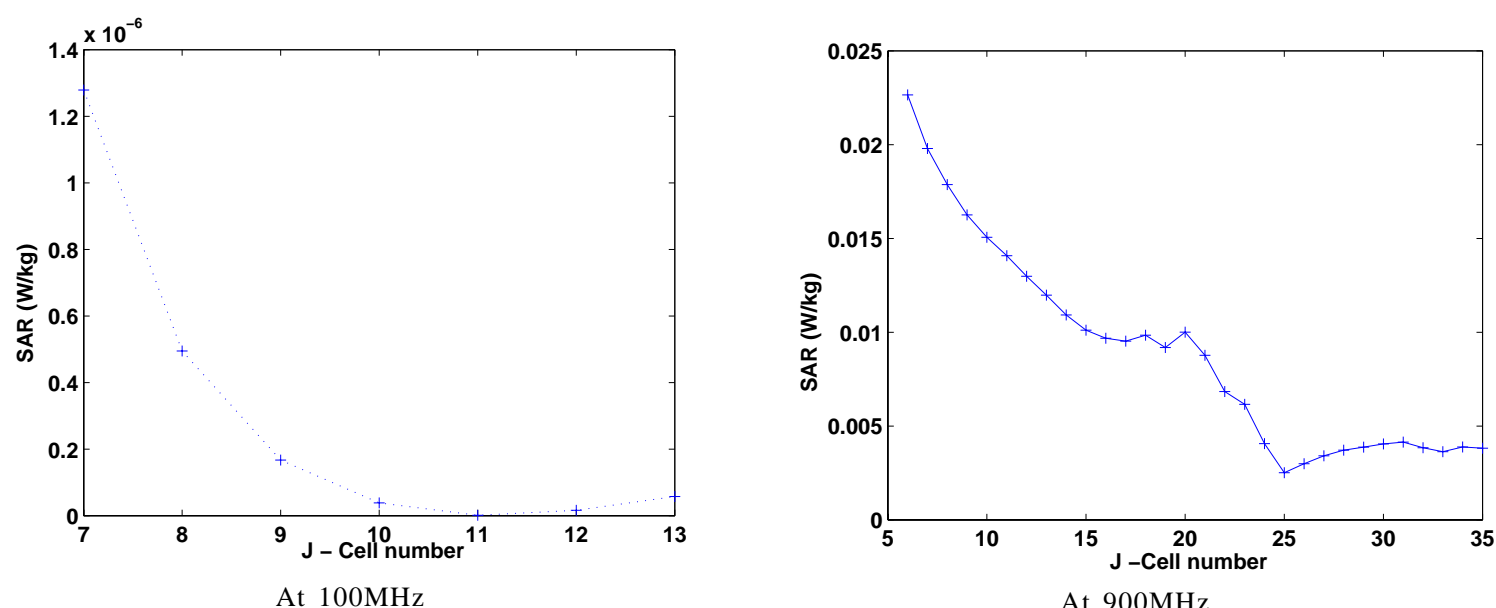

Fig. 4 SAR in the eye
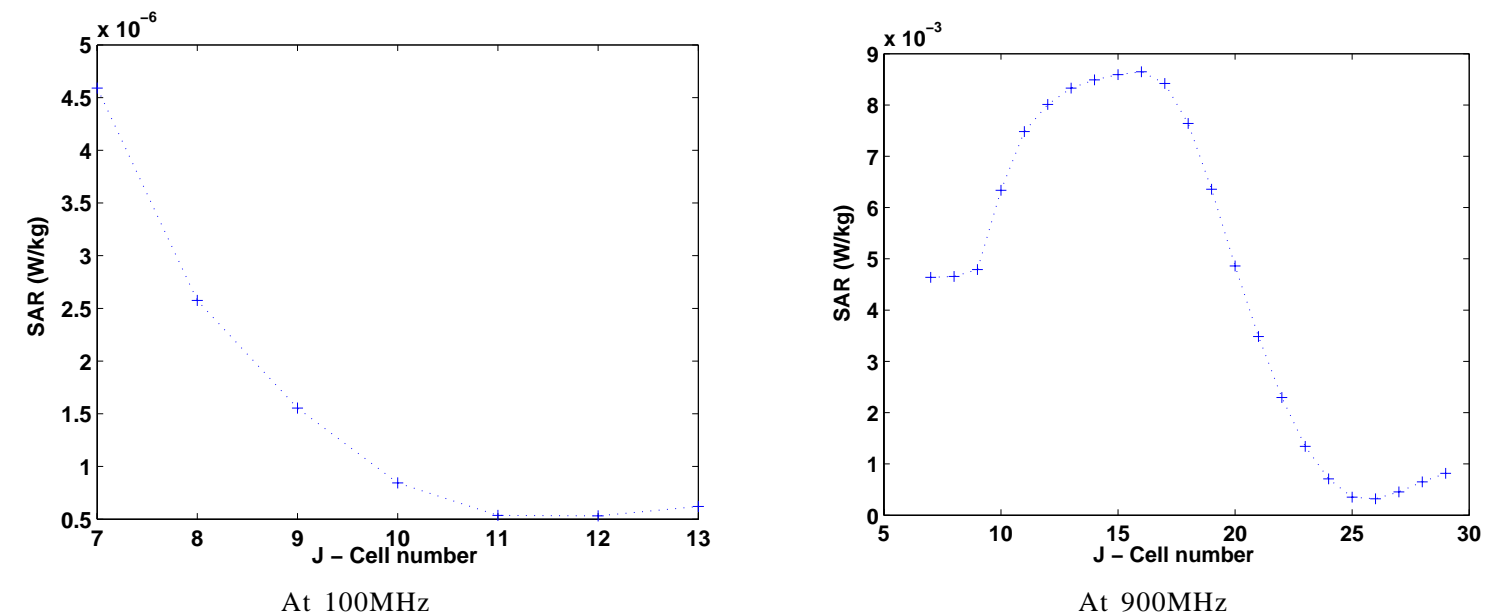

Fig. 5 SAR in the kidney

\subsection{SAR distribution in human body organs}

The distributions of SAR in the four organs, eye, brain, kidney and the testis due to RF and microwave signals of different frequencies is examined at a field strength of $1 \mathrm{~V} / \mathrm{m}$. Calculations are carried out at from $100 \mathrm{MHz}$ to $2500 \mathrm{MHz}$. From $100 \mathrm{MHz}$ to $900 \mathrm{MHz}$, there are some differences in the shape of the SAR distribution curves in the cases of kidney and testis. For clarity, Figs. 4, 5, 6 and 7 show the SAR distributions in the eye, brain, kidney and the testis at $100 \mathrm{MHz}$ and $900 \mathrm{MHz}$. The horizontal axes show the depth of each organ in the direction of propagation of the electromagnetic wave or the signal. The origin corresponds to the centre of the front layer of the organ.

Fig. 6 indicates that the highest SAR values are observed at the front layers of the brain at all frequencies and an increase in the frequency does not affect the shape of the curve. In the case of the kidney, at low frequencies, the highest SAR is observed at the front layers and this gets shifted towards the center of the kidney at $900 \mathrm{MHz}$. 


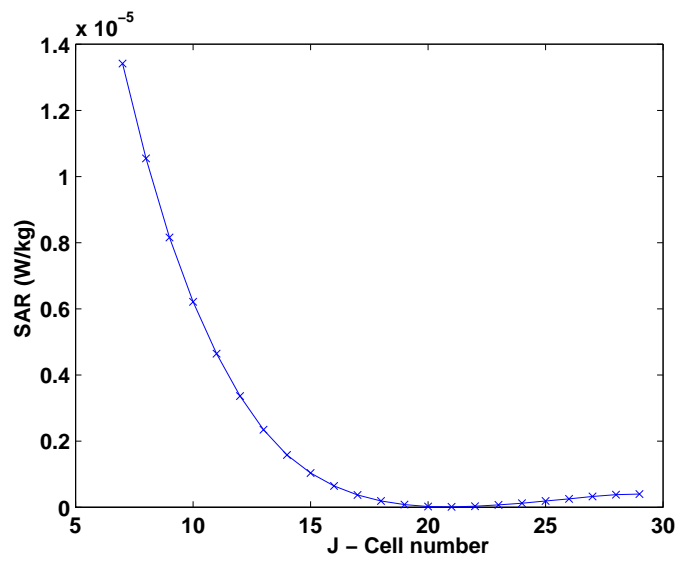

At $100 \mathrm{MHz}$

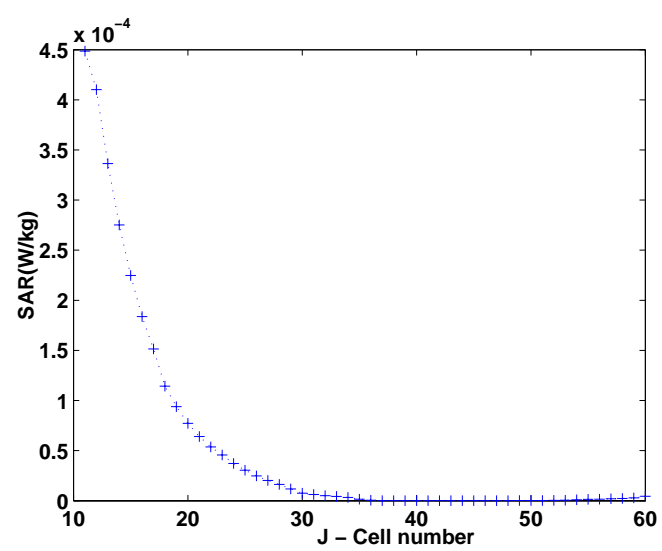

At $900 \mathrm{MHz}$

Fig. 6 SAR in the brain

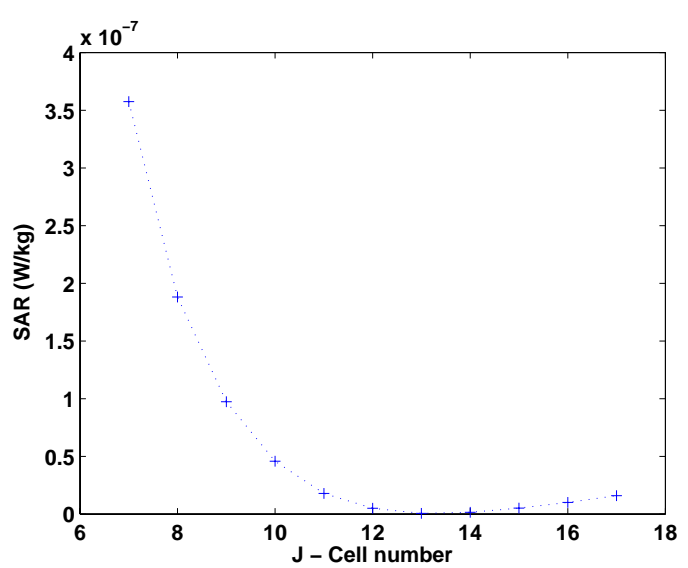

At $100 \mathrm{MHz}$

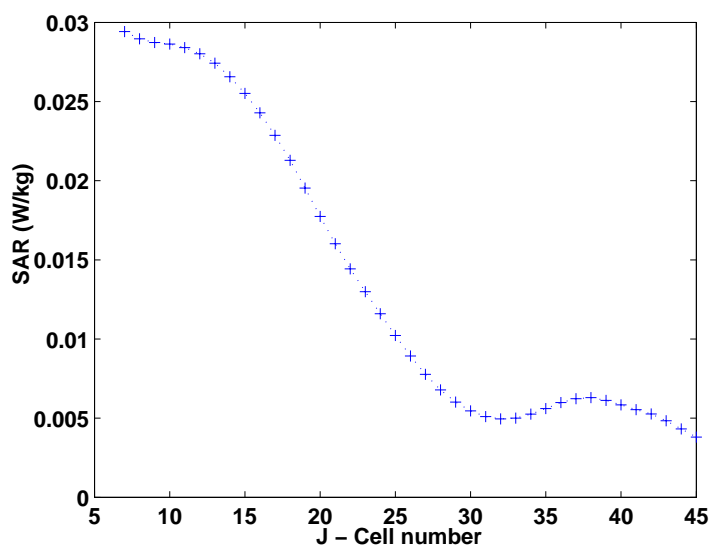

At $900 \mathrm{MHz}$

Fig. 7 SAR in the testis

When the frequency of the incident signal is increased beyond $900 \mathrm{MHz}$, in the eye and the testis, an increase in the SAR value is observed towards the interior of the organ. The eye and testis are said to be more sensitive to higher frequencies [21]. In the case of the eye, the position of the highest SAR gets shifted towards the center of the eye as the frequency increases. This implies that when the eye is exposed to higher frequencies, 'hot spots' are created towards its center as seen in Fig. 8.

A similar behaviour is observed in the testis, as shown in Fig. 9. However the peak value decreases beyond a certain frequency, in this case at around $1800 \mathrm{MHz}$.

In the case of the kidney, the SAR distribution is such that, although at low frequencies the SAR is high at the surface, this value drops to less than half at high frequencies. However, there is a broad peak towards the middle of the testis at high frequencies and the peak becomes narrower and sharper with increasing frequency; its magnitude gradually drops as the frequency increases. This is shown in Fig. 10. Its biological implications are not yet known. 
M. A. A. Karunarathna and I. J. Dayawansa, Sri Lankan Journal of Physics, Vol. 7, (2006), 35-47

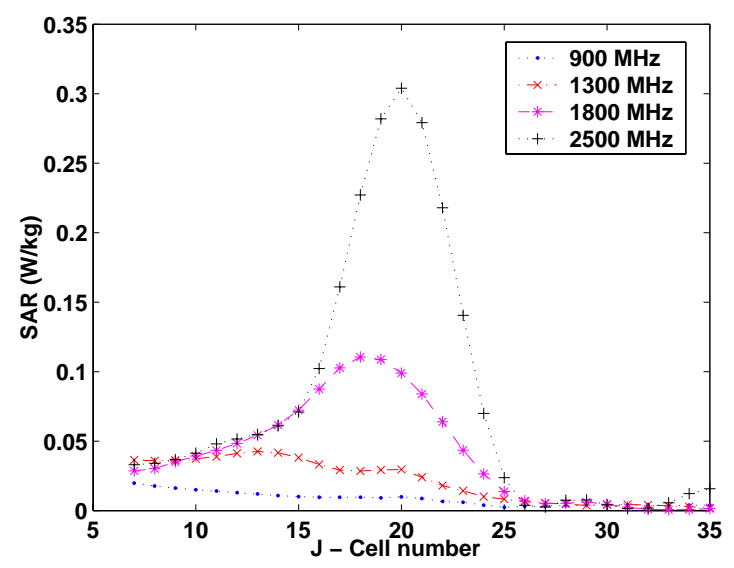

Fig. 8 SAR distribution in the eye

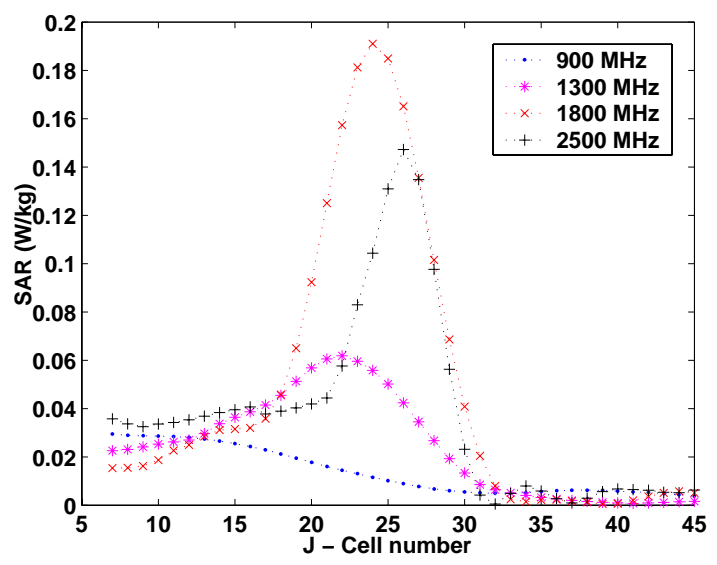

Fig. 9. SAR distribution in the testis

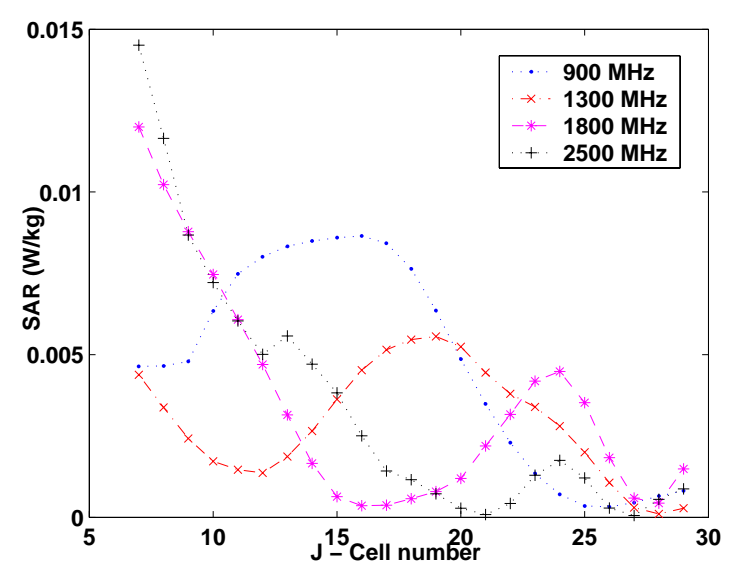

Fig. 10 SAR distribution in the kidney

\section{CONCLUSIONS}

All field measurements taken indicate that the estimated $\mathrm{SAR}_{1 \mathrm{~g}}$ value in the eye tissue of the public, in major cities of Sri Lanka, is reasonably below the FCC standard. However the estimated SAR values are not too far away from safety standards. Our measurements were confined to FM radio, TV and Cellular Mobile operating in the $900 \mathrm{MHz}$ band. We could not take field measurements from CDMA fixed wireless networks operating in the $2500 \mathrm{MHz}$ band, as they went into operation recently. The major contribution to the SAR is from the mobile cellular base station emissions.

For theoretical calculations of SAR in the several organs of the body, we considered signals of frequencies ranging up to $2500 \mathrm{MHz}$. However calculations are based on an incident field of strength of $1 \mathrm{~V} / \mathrm{m}$ which is close to the worst case considering the measured field strengths. It is also the field strength considered by many other researchers. Of the four vital body organs examined, the highest SAR levels are seen to be at the front layers of the organs up to a frequency of $900 \mathrm{MHz}$ except in the case of the kidney. A peak occurs in the SAR in the middle of the eye and in the testis at high 
frequencies. The similar peak in the SAR curve is seen in the kidney. Its magnitude gets smaller when the frequency goes beyond a certain high frequency and the peak gets shifted to the interior with increasing frequency. However, all estimated values are reasonably less than the public safety limits specified by FCC.

When the $3 \mathrm{G}$ mobile communication systems become operational in the very near future, public would be exposed to more microwaves and the exposure levels would go up. The eye and testis would be exposed to higher frequencies such as $3500 \mathrm{MHz}$ and beyond. As such 'hot spots' would be created close to the center of the eye and the testis; this certainly should be of public concern. As the demand for telephone and broadband internet access increases, the wireless network providers would install more base stations with the result that the strength of the field exposed to, by the public, would go up and with that the SAR would go up. Hence it is advisable to carry out SAR evaluations periodically to check and maintain SAR at acceptable levels for public safety. Our results indicate that the maximum SAR values inside the four vital human body organs analyzed in this paper are below FCC standards and therefore at present we are safe.

\section{ACKNOLEDGEMENTS}

The authors are grateful to the Telecommunication Regulatory Commission for the assistance extended in order to carry out calculations.

\section{REFERENCES}

1. IEEE standard for safety levels with respect to human exposure to radio frequency electromagnetic fields $3 \mathrm{kHz}$ to $300 \mathrm{GHz}$, IEEE Std. C95.1, (Institute of Electrical and Electronics Engineers, New York, NY, 1999).

2. Evaluating Compliance with FCC Guidelines for human exposure to Radio Frequency Electromagnetic Fields, FCC OET Bulletin.65, Washington D.C., August 1997.

3. Biological Effects and Exposure Criteria for Radio Frequency Electromagnetic Fields, NCRP Report 86, (National Council on Radiation Protection and Measurements, Bethesda, MD,1986), 171-174.

4. ICNIRP, Guidelines for Limiting Exposure to Time-Varying Electric, Magnetic and Electromagnetic Fields (up to $300 \mathrm{GHz}$ ), Health Physics, 74, (1998), 494-522.

5. NRPB, Health Effects from RF Electromagnetic fields, Report of an independent Advisory Group on Nonionizing Radiation, 14, (2003) 2.

6. James C. Lin, Biological Bases of Current Guidelines for Human Exposure to Radio-Frequency Radiation, IEEE Antennas and Propagation Magazine, $\underline{45}$, (2003) 3.

7. M. A. A. Karunarathna and I. J. Dayawansa, Human Exposure to RF radiation in Sri Lanka, Sri Lankan Journal of Physics, $\underline{6}$, (2005) 19.

8. R. J. Spiegel, A review of numerical models for predicting the energy deposition and resultant thermal response of humans exposed electromagnetic fields, IEEE Trans. Microwave Theory Tech., 23, (1975) 623. 
M. A. A. Karunarathna and I. J. Dayawansa, Sri Lankan Journal of Physics, Vol. 7, (2006), 35-47

9. K. S. Yee, Numerical solution of initial boundary value problems involving Maxwell's equations in isotropic media, IEEE Trans. Antenna Propagat., 17, (1966) 585.

10. A. Taflove and M. E. Morris, Computation of the electromagnetic fields and induced temperatures within a model of the microwave irradiated human eye, IEEE Trans. on Microwave Theory Tech., 23, (1975) 660.

11. K.Umashankar and A. Taflove, A novel method to analyze electromagnetic scattering of complex objects, IEEE Trans. Electromagn. Compat., 24, (1982) 397.

12. A. Taflove and K. Umashankar, Radar cross section of general three-dimensional scatterers," IEEE Trans. Electromagn. Compat., 25, (1983) 433.

13. Dennis M. Sullivan, O. M P. Gandhi and Allen Taflove, Use of the Finite-Difference Time-Domain Method for Calculating EM Absorption in Man Models, IEEE Trans. on Biomedical Engineering, 35, (1988) 3.

14. Dennis M. Sullivan, David T. Borup and O. M. P. Gandhi, Use of Finite-Difference Time-Domain Method in Calculating EM Absorption in Human Tissues, IEEE Trans. on Biomedical Engineering, 34, (1987)2.

15. Hsing-Yi Chen, Hou-Hwa Wang, Current and SAR Induced in a Human Head Model by the Electromagnetic Fields Irradiated from a Cellular Phone, IEEE Trans. on Microwave Theory Tech., 42, (1994) 12.

16. A. Bayliss and E. Turkel, Radiation boundary conditions for wave like equations, Commun. Pure Appl.

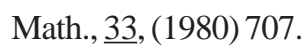

17. Matthew N.O. Sadiku, Numerical Techniques in Electromagnetics. (Florida: CRC Press 1992) 179.

18. Allen Taflove, Morris E. Brodwin, Computation of the Electromagnetic Fields and Induced Temperatures Within a Model of the Microwave-Irradiated Human Eye, IEEE Trans. on Microwave Theory Tech., 23, (1975) 888.

19 Harold Ellis, Clinical Anatomy - A revision and applied anatomy for clinical students, (Blackwell scientific publications, London, 1980).

20. C. Gabriel, Compilation of the dielectric properties of body tissues at RF and microwave frequencies, Tech. Rep. AL/OE-TR-(1996) (Brooks Air Force, Brooks AFB, TX, 1996).

21. P. Bernardi, M. Cavagnaro, S. Pisa and E. Piuzzi, Human exposure to radio base station antennas in urban environment, IEEE Trans.Microwave Theory Tech., 48, (2000) 1996.

22. J. C. Lin, Cataracts and Cell-Phone Radiation, IEEE Antennas and Propagation Magazine, 45, (2003) 171.

23. IEEE Recommended Practice for Measurements and Computations of Radio Frequency Electromagnetic Fields With Respect to Human Exposure to Such Fields, 100 kHz-300 GHz, IEEE Std. C95.3, (IEEE, New York, 2002). 